\title{
BMJ Open Cost-effectiveness of oral anticancer drugs and associated individualised dosing approaches in patients with cancer: protocol for a systematic review
}

\author{
Madelé van Dyk (D) , ${ }^{1,2}$ Norma Bulamu, ${ }^{2,3}$ Chelsea Boylan, ${ }^{1,2}$ \\ Anna M Mc Laughlin (D) , ${ }^{4,5}$ Ganessan Kichenadasse, ${ }^{1,6}$ Nikki May, ${ }^{7}$ \\ Robin Michelet, ${ }^{4,8}$ Charlotte Kloft, ${ }^{4,8}$ Billingsley Kaambwa ${ }^{3}$
}

To cite: van Dyk M, Bulamu N, Boylan C, et al. Cost-effectiveness of oral anticancer drugs and associated individualised dosing approaches in patients with cancer: protocol for a systematic review. BMJ Open 2021;11:e047173. doi:10.1136/ bmjopen-2020-047173

- Prepublication history and additional supplemental material for this paper are available online. To view these files, please visit the journal online. (http://dx.doi.org/10.1136/ bmjopen-2020-047173)

Received 22 November 2020 Accepted 27 July 2021

Check for updates

(C) Author(s) (or their employer(s)) 2021. Re-use permitted under CC BY-NC. No commercial re-use. See rights and permissions. Published by BMJ.

For numbered affiliations see end of article.

Correspondence to Dr Madelé van Dyk; madele.vandyk@flinders.edu.au

\section{ABSTRACT}

Introduction Oral anticancer drugs (OADs) have rapidly expanded with more than 70 OADs targeting several molecular targets. Many of the OADs exert an exposureresponse relationship but still, a 'one-size fits-all' dose is used, ignoring interindividual variability. Several of these OADs share similar mechanisms of actions and thus target the same cancer and has resulted in a substantial research focus on comparing the health benefit of each. However, significantly less is known about the cost-benefit associated with OADs. This paper will provide a protocol to systematically review studies that have evaluated the costeffectiveness of OADs and their associated individualised dosing interventions.

Methods and analysis Systematic review methodology will be applied to identify, select and extract data from published economic evaluation (costs and outcomes/ benefits) studies of OADs and their associated individualised dosing interventions. Bibliographic databases (eg, Ovid EMBASE, Ovid MEDLINE) will be used to perform the systematic literature search (between 1 January 2000 and October 2020). Only full economic evaluations will be included, but no restrictions on study outcomes will be applied. The quality of included primary studies will be assessed using the Consolidated Health Economic Evaluation Reporting Standards checklist for reporting economic evaluations. Studies with low-quality evidence will be excluded. A narrative synthesis of the results from the included studies will be undertaken, with a subgroup analysis where appropriate.

Ethics and dissemination This systematic review will not require ethics approval as there will not be any collection of primary data. Findings of this review will be disseminated through publications in peer-reviewed journals, presentations at workshops or conferences and sharing through a media release. Findings from this review will provide evidence to direct and inform policy-makers where cost-neutral strategies may be effective or where dose individualising strategies may be economically beneficial. Additionally, gaps will be identified in the current literature to inform future-related research. PROSPERO registration number CRD42020218170. Electronic supplemental material The online version of this article contains supplemental material, which is available to authorised users.
Strengths and limitations of this study

- In the past two decades, the clinical use of targeted therapies has increased significantly, and high costs are associated with these drugs; therefore, this systematic literature review will be used for up-to-date cost-effectiveness analyses on all oral anticancer drugs (OADs).

- A big focus has been put on 'precision medicine', but very little focus has been put on 'precision dosing', therefore, this study will be the first to summarise available costs associated with all dose individualising strategies for all OADs.

- Since most economic evaluations exclude lowincome and middle-income countries, our study will include data from such countries in a subgroup analyses (separate to the high-income countries) to reflect a global and more realistic cost representation.

- Our study only focuses on OADs since the use has significantly increased, however, a large proportion of patients still undergo chemotherapies, which our study does not include (unless it was compared with an OAD).

\section{INTRODUCTION}

Since the introduction of several targeted therapies such as small molecule kinase inhibitors (KIs) and monoclonal antibodies, treatment options have increased, and have led to improved survival outcomes. ${ }^{12}$ The majority of these targeted therapies are oral anticancer drugs (OADs) with more than 70 European Medicines Agency and/or US Food and Drug Administration-approved OADs. ${ }^{2}$ Not only have OADs reshaped the convenience of how we administer anticancer treatments and thus avoid the need for repeated hospital visits for parenteral treatment, reducing healthcare costs, but they also have superior survival benefit compared with the conventional and cytotoxic chemotherapies. ${ }^{2}$ At the same time, associated costs in non-elderly patients 
have increased by more than factor two from US $\$ 3381$ patient per month in 2001 to US\$7370 in 2011 and will have continued to increase since then ${ }^{4}$ and the economic impact of cancer costs is significant. ${ }^{5}$ OADs mainly consist of small molecule inhibitors such as KIs, poly adenosine diphosphate-ribose polymerase (PARP) inhibitors (PARPi) and isocitrate dehydrogenase inhibitors (IDHIs). Hormone drugs (HDs) are also OADs but have been used for several decades ${ }^{6}$ with only a few newer drugs released in the past decade. However, for the inhibitors such as the KIs, many next-generation drugs have been approved for the same cancers in the past two decades, resulting in many treatment options available and subsequently, an attraction in research studies comparing health and costbenefit of each, with the latter being the least studied. ${ }^{7}$ High costs are associated with targeted OADs; therefore, economic evaluations are critical. ${ }^{2}$ Currently, to the best of our knowledge, no up-to-date systematic review exists that focuses on the cost-effectiveness of OADs.

A well-known disadvantage of most anticancer drugs is the wide interindividual variability in patient responses, because of the variability in their pharmacokinetic properties, that is, absorption, distribution, metabolism and elimination. ${ }^{1}$ In the case of OADs, variability is further complicated by the absorption phase due to passing through the gastrointestinal tract, which intravenously given drugs do not have. ${ }^{13}$ Typically, with many of these targeted therapies, a strong focus on "precision medicine' has been observed, using, for example, genetics for optimal drug selection ('the what': what to give). However, despite wide variability in pharmacokinetics resulting in wide variability in the amount of the drug concentrations in the blood, no strategy is used for optimal dose selection ('the how': how much drug, how often, how long). ${ }^{89}$ Several OADs have a clear concentration-response relationship, meaning the amount of drug in the blood relates to how the individual will respond. Despite, knowing this, the majority of OADs are currently dosed with a 'one-size-fits-all' approach, which means irrespective of each patient's individual characteristics, they will receive the same dose. Therefore, it is no surprise that such large variability in response is observed. ${ }^{189}$ This large variability in response has major clinical implications and results in suboptimal outcomes with patients experiencing serious adverse events or therapeutic failure due to underdosing or overdosing, respectively. This wide variability in patient responses is a well-recognised clinical problem and has resulted in many 'individualised dosing' strategies. Such strategies typically include therapeutic drug monitoring (TDM), toxicity-adjusted dosing (TAD), model-informed precision dosing (MIPD), genotyping or phenotyping approaches. ${ }^{1}$ Although significant evidence exists to support precision dosing, the broader clinical uptake of precision dosing is hindered by the apparent preclusive cost and complexity of generating sufficiently powered evidence to support the clinical validity of the benefit. It has been suggested that this approach is not currently implemented due to high costs. ${ }^{1}$ However, the economic healthcare costs have not been assessed in the real-world setting to date. As such, significant research effort is required to address the cost-effectiveness of OADs in order to evaluate the true cost associated with their use and to identify the most expensive drugs when health benefit is incorporated. This will direct where costneutral strategies may be effective or where dose individualising strategies may not only improve the health benefit but also the cost-benefit. Therefore, this paper will provide a protocol to systematically review economic evaluation studies that have assessed the costs and benefits of OADs and finding evidence to improve efficiency through dose tailoring.

\section{RESEARCH AIMS AND OBJECTIVES}

This study aims to systematically review evidence on the cost-effectiveness of (1) OADs when compared directly with other anticancer treatments for the same cancers and (2) individualised dosing interventions when compared with standard dosing. Secondary objectives include assessing the evidence and limitations around the methods of cost-effectiveness analyses, as well as the variation of outcomes and costs included between interventions, settings and countries.

\section{METHODS AND ANALYSIS}

\section{Patient and public involvement}

There will be no patient or public participation involvement as this systematic review is capturing previous findings. However, we would like to acknowledge Mr Ryan Hodges, from our consumer engagement group who have provided verbal feedback on our study design for this protocol paper and will continue that into the systematic literature review too.

\section{Inclusion criteria}

- Studies comparing OADs directly with other anticancer treatments for the same cancers or studies comparing individualised dosing interventions (in cancer) to standard dosing.

- Full economic evaluation studies (where both costs and outcomes are compared).

- Trial-based or non-trial-based studies.

- Descriptive, quantitative or simulation-based studies.

- Studies published in the English language.

Depending on the type of economic evaluation, measures of outcome differ. For example, quality-adjusted life-years (QALYs) are the standard outcome measures in cost utility analysis, monetary outcomes in cost-benefit analysis while clinical outcomes such as survival are used in cost-effectiveness studies. ${ }^{10}$ All types of economic evaluations will be included. Studies applying either QALYs, monetary or clinical outcomes as a measure of effectiveness will be included. Studies undertaken in low-income and middle-income countries (LMICs) will be included as a subgroup analyses (separate to the high-income 
countries) where appropriate due to inherent differences in healthcare systems pricing and funding. This is a pragmatic approach because the percentage of gross domestic product allocated to healthcare in developed nations such as the UK, Spain, Australia is approximately $9 \%-12 \%$, compared with $4 \%-6 \%$ in LMICs.

\section{Exclusion criterion}

- Studies that do not investigate both costs and outcomes in OADs.

- Economic evaluations of individualised dosing interventions of OADs prescribed for supportive cancer treatments (eg, antimicrobials and steroids).

The reviewers will extract relevant data on study design, population, intervention(s), outcome(s) and effect size(s). These data will be used for the quality assessment of the review.

\section{Condition or domain}

Condition or domain under the study is cancer.

\section{Population}

Real or simulated individuals of any gender and ethnicity, inpatients or outpatients, who are on OADs.

\section{Cost and outcome measures}

There will be no restrictions on study outcomes because the purpose of the review is to assess all outcomes reported within economic evaluations of OADs. Relevant primary outcomes will include disability-adjusted life-years, QALYs, perceived quality of life/health status measurements and/or clinical outcomes such as patient survival, treatment response, patient cure rate, duration of treatment, adherence, estimation of adverse events after a follow-up period of 6-12 weeks (and longer if available), hospitalisations and change of drug prescribed/ treatment over time for the same cancers.

\section{Exposures/interventions}

The primary exposure in this review will be any OADs (KIs, HDs, PARPIs, IDHIs) used for the treatment of cancer in adult patients. Any intervention aimed at individualising drug dosage such OADs will also be included. Considering that treatment and individualised dose strategies/ interventions are likely to be diverse, the findings will be synthesised separately for all types of interventions (TDM, TAD, MIPD, genotyping or phenotyping approaches).

\section{Study design}

The systematic review will consider quantitative studies of good quality, as outlined below, published between 1 January 2000 and October 2020. The searches will be rerun just before the final analyses and further studies retrieved for inclusion.

\section{Search strategies}

The following steps will be undertaken to perform the search strategy. As well as addressing the objectives stated above, this review will also update a previous review conducted by Smieliauskas $e t a l^{7}$ and their search strategy will be updated to include the concepts below. First, we will search Google scholar and MEDLINE (PubMed) in order to develop key terms for the pre-defined concepts relating to the research question:

- Concept 1: will look at the health economics terms such as cost-effectiveness, cost-benefit, cost-utility, economic evaluation and QALYs.

- Concept 2: will focus on the disease area with terms such as cancer and neoplasms

- Concept 3: will be interventions being compared, that is, orally administered drugs with terms related to the actual drugs in this group

Concepts 1, 2 and 3 will be connected by 'AND' to run the full search strategy. A detailed search strategy applied in Medline is provided in online supplemental appendix 1.

Second, we will carry out a full search using all identified keywords and index terms across the following databases: Ovid Embase, Ovid MEDLINE, Ovid Cochrane, EBSCO CINAHL, Ovid Econlit, SCOPUS, Web of Science, Ovid EmCare and NHS EED. Finally, we will undertake backward and forward citation chaining of relevant documents.

\section{Study selection}

Titles and abstracts from each database will be screened and relevant records selected for a full-text appraisal. The study selection process will follow the Preferred Reporting Items for Systematic Reviews and Meta-Analyses guidelines. ${ }^{11}$ Search results will be exported into a citation management software, EndNote and into the systematic review software Covidence. Titles and abstracts will be distributed among six independent reviewers (researchers in life sciences/health economics and healthcare professionals), for screening against the inclusion criteria, with $15 \%$ assigned to all reviewers. The strength of agreement between reviewers will be estimated by calculating the intraclass correlation coefficient. ${ }^{12}$ Two reviewers will then assess the full text of selected articles for eligibility against the inclusion criteria. Any disagreement or conflicting views between the reviewers over the eligibility of specific studies will be resolved by discussion or the final judgement of a third reviewer. Included articles will then progress to quality assessment or critical appraisal, data extraction and analysis. Both stages of the selection process will be piloted and if necessary modified.

\section{Quality assessment}

Quality of studies will be assessed using the Consolidated Health Economic Evaluation Reporting Standards tool/checklist for reporting economic evaluations. ${ }^{13}$ The Checklist contains 24 items, each item given a 1 or 0 depending on whether it is achieved or not in the study. Studies with fewer than 20 out of 24 will be considered to contain a high risk of bias and therefore will not be included in the final analysis. In addition, modelling studies will be assessed using the 'Good Practice 
Guidelines for Decision-Analytic Modelling in Health Technology Assessment'. ${ }^{14}$ Studies with low-quality evidence will be excluded. The transferability of results will also be assessed using the European Network of Health Economic Evaluation Databases transferability checklist. ${ }^{15}$ The scoring of the papers will be divided into four categories: poor quality (scoring 40\%-55\%), good quality (scoring 55\%-70\%), very good quality (scoring 70\%-85\%) and excellent quality (scoring $85 \%$ or higher). To validate the quality assessment process, the process will be independently checked for completeness and accuracy by a third reviewer.

\section{Analysis of subgroups or subsets}

Subgroup analysis will be conducted for studies comparing the same $\mathrm{OAD}$ or intervention, same disease and within the same healthcare system.

\section{Data extraction}

Quantitative data will be extracted from papers included in the review using the standardised Joanna Briggs Institute (JBI) data extraction tool for economic evaluations. ${ }^{16}{ }^{17}$ The JBI is an independent, international, notfor-profit research and development organisation that develops critical appraisal checklists for qualitative and qualitative research including economic evaluations of healthcare interventions.

The data extracted will include specific details about the intervention and comparators, the settings, the population and sample size, costs and outcomes as well as details of the results. This tool will be modified to include any other information that is relevant for this study. A copy of the tool is provided in online supplemental appendix 2. Whenever, there is missing or unclear data, we will contact the authors of primary studies. If no response is received, interpolation, digitising and citing articles will be explored. Primary reviewers will independently check the data extraction. The information to be extracted will include that on the study population and setting, intervention and comparator, economic evaluation methods, analytic perspective(s), source of effectiveness data, prices and currency, analytical duration, cost-effectiveness method, sensitivity analysis, measures of resource use, cost and health outcome(s) as well as summary of the cost-effective analysis (CEA) results, for example, incremental cost-effectiveness ratios.

\section{Strategy for data synthesis}

Following data extraction, the reviewers will provide a qualitative narrative synthesis of the results from the included studies, structured around general characteristics, characteristics of the intervention programmes and economic findings (basic characteristics, study perspectives, resource use, cost categories, cost-effectiveness findings and sensitivity analysis), along with a critique of methods used for economic evaluation. The emphasis of the analysis will be on the cost-effectiveness of the interventions compared, the methodology applied, detail of derivation and adjustment of costs and outcomes for different times, and applicability of results to other healthcare settings. Cost related inputs will be converted to US\$ (2020) for ease of comparison. For model based economic evaluation studies comparing the same interventions, the health states included, measures of effectiveness, levels of effectiveness and other model parameters will be extracted and compared directly to other studies. Model designs will also be compared with discuss limitations of study results as well as the sensitivity analysis.

\section{Ethics and dissemination}

This systematic review will not require ethics approval as there will not be any collection of primary data. Therefore, no participant consent will not be required. Findings of this review will be disseminated through publications in peer-reviewed journals, presentations at workshops or conferences and sharing through a media release.

\section{CONCLUSION}

This systematic review will provide evidence of costeffectiveness support of, or against the hypothesis that some OADs are value-for-money compared with others, and whether individualised dosing of OADs improves patient outcomes at a lower cost due to a decrease in noncompliance and/or a reduced rate of adverse events, or therapeutic failure.

\section{Author affiliations}

${ }^{1}$ Flinders Health \& Medical Research Institute- Cancer, Flinders University College of Medicine and Public Health, Bedford Park, South Australia, Australia

${ }^{2}$ Flinders Centre for Innovation in Cancer, Flinders University College of Medicine and Public Health, Bedford Park, South Australia, Australia

${ }^{3}$ Health Economics, Flinders University College of Medicine and Public Health, Bedford Park, South Australia, Australia

${ }^{4}$ Department of Clinical Pharmacy and Biochemistry, Institute of Pharmacy, Freie Universität Berlin, Berlin, Germany

${ }^{5}$ Postdam/Berlin, PharMetrX Graduate Research Training Program, Postdam/Berlin, Germany

${ }^{6}$ Medical Oncology, Flinders Medical Centre, Bedford Park, South Australia, Australia ${ }^{7}$ SA Health Library Service, Bedford Park, South Australia, Australia

${ }^{8}$ PharMetrX Graduate Research Training Program, Postdam/Berlin, Germany

Contributors Conception or design: MvD, NB, BK, RM and NM. Acquisition or analysis of data: MvD, NB, CB, AMML and Meuller-Schoell. Interpretation of Data: $\mathrm{MvD}, \mathrm{NB}, \mathrm{CB}$ and GK. Drafting the work or revising for intellectual content: MvD, NB, $\mathrm{CB}$, Meuller-Schoell, GK, NM, RM, CK and BK. Final approval of the version to be published: MvD, NB, CB, Meuller-Schoell, GK, NM, RM, CK and BK. Agreement to be accountable for all aspects of the work: MvD, NB, CB, Meuller-Schoell, GK, NM, RM, $\mathrm{CK}$ and $\mathrm{BK}$.

Funding MvD reports grants from Cancer Council SA/Flinders University (ECR Beat Cancer/10686). CK reports grants from an Industry Consortium (AbbVie Deutschland KG, AstraZeneca, Boehringer Ingelheim Pharma KG, Grünenthal, F. Hoffmann-La Roche Ltd, Merck KGaA and SANOFI) for the PharMetrX program/Grant Number: NA; The Federal Ministry of Education/ Grant Number: NA and Research and the European Commission within in the Horizon 2020 framework programme Grant Number: NA. All other authors have nothing to disclose.

Competing interests None declared.

Patient consent for publication Not required.

Provenance and peer review Not commissioned; externally peer reviewed.

Supplemental material This content has been supplied by the author(s). It has not been vetted by BMJ Publishing Group Limited (BMJ) and may not have been 
peer-reviewed. Any opinions or recommendations discussed are solely those of the author(s) and are not endorsed by BMJ. BMJ disclaims all liability and responsibility arising from any reliance placed on the content. Where the content includes any translated material, BMJ does not warrant the accuracy and reliability of the translations (including but not limited to local regulations, clinical guidelines, terminology, drug names and drug dosages), and is not responsible for any error and/or omissions arising from translation and adaptation or otherwise.

Open access This is an open access article distributed in accordance with the Creative Commons Attribution Non Commercial (CC BY-NC 4.0) license, which permits others to distribute, remix, adapt, build upon this work non-commercially, and license their derivative works on different terms, provided the original work is properly cited, appropriate credit is given, any changes made indicated, and the use is non-commercial. See: http://creativecommons.org/licenses/by-nc/4.0/.

\section{ORCID iDs}

Madelé van Dyk http://orcid.org/0000-0001-8721-6370

Anna M Mc Laughlin http://orcid.org/0000-0002-5936-1877

\section{REFERENCES}

1 Rowland A, van Dyk M, Mangoni AA, et al. Kinase inhibitor pharmacokinetics: comprehensive summary and roadmap for addressing inter-individual variability in exposure. Expert Opin Drug Metab Toxicol 2017;13:31-49.

2 Mueller-Schoell A, Groenland SL, Scherf-Clavel O, et al. Therapeutic drug monitoring of oral targeted antineoplastic drugs. Eur J Clin Pharmacol 2021;77:441-64.

3 Schlichtig K, Dürr P, Dörje F, et al. New oral anti-cancer drugs and medication safety. Dtsch Arztebl Int 2019;116:775-82.

4 Deloitte. The economic cost of cancer in adolescents and young adults, 2018. Available: https://www2.deloitte.com/au/en/pages/ economics/articles/economics-cost-cancer-adolescents-youngadults.html

5 Shih Y-CT, Smieliauskas F, Geynisman DM, et al. Trends in the cost and use of targeted cancer therapies for the privately insured Nonelderly: 2001 to 2011. J Clin Oncol 2015;33:2190-6.
6 Early Breast Cancer Trialists' Collaborative Group. Effects of adjuvant tamoxifen and of cytotoxic therapy on mortality in early breast cancer. N Engl J Med Overseas Ed 1988;319:1681-92.

7 Smieliauskas F, Chien C-R, Shen C, et al. Cost-Effectiveness analyses of targeted oral anti-cancer drugs: a systematic review. Pharmacoeconomics 2014;32:651-80.

8 Di Gion P, Kanefendt F, Lindauer A, et al. Clinical pharmacokinetics of tyrosine kinase inhibitors. Clin Pharmacokinet 2011;50:551-603.

9 van Erp NP, Gelderblom H, Guchelaar H-J. Clinical pharmacokinetics of tyrosine kinase inhibitors. Cancer Treat Rev 2009;35:692-706.

10 Drummond MF, Sculpher MJ, Torrance GW, et al. Methods for the economic evaluation of health care programs. Oxford: Oxford University Press, 2005

11 Liberati A, Altman DG, Tetzlaff J, et al. The PRISMA statement for reporting systematic reviews and meta-analyses of studies that evaluate healthcare interventions: explanation and elaboration. BMJ 2009;339:b2700.

12 Rosner BA, ed. The intraclass correlation coefficient. Pacific Grove: Fundamentals of biostatistics, 2011.

13 Husereau D, Drummond M, Petrou S, et al. Consolidated health economic evaluation reporting standards (cheers) statement. BMJ 2013;346:f1049.

14 Philips Z, Bojke L, Sculpher M, et al. Good practice guidelines for decision-analytic modelling in health technology assessment: a review and consolidation of quality assessment. Pharmacoeconomics 2006;24:355-71. doi:10.2165/00019053200624040-00006

15 Nixon J, Rice S, Drummond M, et al. Guidelines for completing the EURONHEED transferability information checklists. Eur $J$ Health Econ 2009;10:157-65.

16 The Joanna Briggs Institute. Joanna Briggs Institute reviewers' manual: 2014 edition/supplement. Adelaide, Australia: The Joanna Briggs Institute, 2014. https://drive.google.com/file/d/12OkTDHv Z9mGxGHKfsallOT563Bffom-t/view

17 Ma L-L, Wang Y-Y, Yang Z-H, et al. Methodological quality (risk of bias) assessment tools for primary and secondary medical studies: what are they and which is better? Mil Med Res 2020;7:7. 